\title{
6 Sprachliche Konstruktionen zwischen Produkt, Prozess und Sprachwissen
}

Theoretische Überlegungen und empirische Erkenntnisse aus Spracherwerbsstudien, die von einem dynamischen Sprachkonzept und einem gebrauchsbasierten Ansatz ausgehen, stehen im Zentrum des folgenden Kapitels. Es stützt sich auf das konstruktionsgrammatische Paradigma, das als Sammelbegriff verschiedene forschungsmethodische Ausrichtungen vereint, die von sogenannten Konstruktionen als grundlegender Einheit von Sprache - und zwar für den Erwerb, die kognitive Repräsentation und für die Verarbeitung gleichermassen - ausgehen. Das folgende Kapitel arbeitet das Potenzial von Konstruktionen als sprachliche Grössen der Sprachaneignung, der sprachlichen Repräsentation und der Sprachproduktion resp. -verarbeitung heraus und zeigt exemplarisch, wie das Konzept auf die schriftliche Sprache und ihre Aneignung übertragen werden kann. Die Ausführungen knüpfen dabei inhaltlich an bereits erwähnte Konzepte, so etwa die Quant-Einheiten (vgl. Kap. 5.3.2), an.

\section{1 Über welche Muster wird Sprache erworben?}

Die Frage, welche sprachlichen Grössen und Muster psycholinguistisch und in der Sprachproduktion und -rezeption relevant sind, stellt eine Grundfrage der Linguistik im Allgemeinen dar. Sie wurde lange insbesondere unter der syntaktischen Musterhaftigkeit verhandelt und bearbeitet, umfasst aber als Grundfrage Konstruktionen unterschiedlicher Art. Die Betonung der Musterhaftigkeit hat auch sprachtheoretisch umfassende Konsequenzen, wie Ágel (2004) ausführt:

Sollte der sprachliche Normalfall nicht das Okkasionelle, sondern das Geprägte und Vorgeprägte sein, müssten Theorien und Methoden der Linguistik darauf umgestellt werden, dass die ad-hoc-Bildungen mehr oder weniger kreative Realisierungen idiomatisch geprägter und vorgeprägter Modelle darstellen, dass also die grammatischen Regeln auf der Basis dieser Modelle funktionieren. (Ágel 2004: 67, zit. nach Feilke 2007: 64).

In den Fokus rücken dadurch diese ,idiomatisch geprägte[n] und vorgeprägte[n] Modelle“ (Ágel 2004: 67, zit. nach Feilke 2007: 64) und ihre Funktion im Spracherwerb und in der Spracheverwendung. Das konstruktionsgrammatische Paradigma, das sich spätestens durch Tomasellos Rezension von Adele Goldbergs Constructions (Goldberg 1995) in der Spracherwerbsforschung etabliert und durch diese an Bekanntheit gewonnen hat (Behrens 2009: 427-428), fasst diese vorgeprägten Strukturen mit jeweils lexikalischen und grammati-

๑ Open Access. (c) 2018 Pascale Schaller, publiziert von De Gruyter. (c) BY-NC-ND Dieses Werk ist lizenziert unter der Creative Commons Attribution-NonCommercial-NoDerivatives 4.0 Lizenz.

https://doi.org/10.1515/9783110555165-006 
schen Anteilen unter dem Terminus Konstruktion zusammen. Das Konzept der Konstruktionen erweist sich als adäquat, um Spracherwerbsprozesse zu beschreiben, auch wenn es ursprünglich nicht als Lerntheorie konzipiert ist. Es wird mit den grundlegenden Annahmen der Kognitiven Linguistik in der Version Langackers „,zur Gebrauchsbasiertheit der Sprache und über die Mechanismen der Generalisierung und Strukturbildung kombiniert (usage-based approach)“ (Behrens 2009: 428, Hervorhebung im Original). Konstruktionen werden als die einzige und grundlegende Einheit für den Erwerb, aber ebenso für die Repräsentation und die Verarbeitung von sprachlichem Wissen angesetzt. Sie stellen Strukturen unterschiedlicher Abstraktionsgrade dar und fügen sich der modularen Trennung von Grammatik und Lexikon nicht (Lasch \& Ziem 2011). Unlängst haben sich konstruktionsgrammatische Ansätze auch in der Spracherwerbsforschung etabliert. Der Konstruktionsbegriff bietet sich in der Spracherwerbsforschung an, um erwerbsrelevante Strukturen von linguistischen Kategorien zu unterscheiden und dadurch der Lernervarietät Rechnung zu tragen. Behrens (2011a: 165) bezeichnet es zu Recht als Kernproblem der Spracherwerbsforschung, dass sie ein sich entwickelndes System untersucht, das oberflächlich der Erwachsenensprache mehr oder weniger ähnlich ist: Es biete sich dadurch an, Kindersprache als Abweichung vom Erwachsenensystem zu beschreiben. Die adäquate Beschreibung der Erwachsenen- und damit der Zielsprache sei notwendig, da „[o]hne Annahmen darüber, wie das Sprachsystem Erwachsener beschaffen und mental repräsentiert ist, [...] das Erwerbsproblem nicht definiert werden [kann]“ (Behrens 2011a: 165). Ebenso wichtig wie Kenntnisse über die Zielvarietät seien allerdings Annahmen über den Ausgangspunkt des Erwerbsprozesses und damit die Frage, welche Fähigkeiten Kinder zum Spracherwerb mitbringen und wie sich diese Erfahrungen in der sprachlichen und nichtsprachlichen Umwelt entwickeln (Behrens 2011a: 165). Die Beschreibung aufgrund der Zielvarietät laufe hingegen Gefahr, kindliche Sprache aus der Defizitperspektive zu modellieren. Konstruktionsgrammatische Ansätze böten hierzu die Alternative, den ,Eigenregeln ${ }^{636}$ der Kindersprache Rechnung zu tragen und diese als Varietät eigener Art zu beschreiben. Der Ausgangspunkt konstruktionsgrammatischer Überlegungen besteht in der Annahme, dass sich Sprachstrukturen aus dem Gebrauch heraus entwickeln, sich mit diesem stetig verändern und dadurch nie ein geschlossenes System etablieren (Ziem \& Lasch 2013: 163). Die konstruktionsgrammatische Analyse der kindlichen Sprachentwicklung stellt damit ein besonders aufschlussreiches Forschungsfeld dar, um nachzuvollziehen, welche Konstruktionen sich im Er-

36 Auf diese ,Eigenregeln“ haben Stern \& Stern (1928) bereits in den 1920er Jahren hinwiesen (Ziem \& Lasch 2013: 163). 
werbsverlauf wie entwickeln. Sie bedient sich dafür zwar in vielen Fällen der Terminologie der meist traditionellen Grammatik, vermeidet aber die Analyse von Kindersprache durch erwachsenensprachliche Kategorien. Ins Zentrum gerückt werden die Forschungsfragen, was Kinder über die entsprechenden Kategorien oder Strukturen wissen und wie produktiv sie sind (Behrens 2011a: 172).

Im Folgenden wird auf den Konstruktionsbegriff eingegangen, indem er zuerst im Allgemeinen und dann bezogen auf die Schreibforschung thematisiert wird. Die Ausführungen münden schliesslich darin, Konstruktionen als Grössen an der Schnittstelle zwischen Sprachwissen, Sprachprozess und Sprachprodukt auszuweisen.

\subsection{Zum Konstruktionsbegriff}

Die Kognitive Linguistik modelliert sprachliche Strukturen als Form-FunktionsAssoziierung: Eine konzeptuelle oder mentale Entität wird durch eine materielle, wahrnehmbare Entität (etwa Laute, Schriftzeichen, Gesten) symbolisiert. Form und Funktion können nicht getrennt werden und sind als sprachliche Einheit, die sie zusammen bilden, mehr oder weniger abstrakt. Sie erreichen ihren Status als Einheit dann, wenn sie als vorgefertigte Einheit kognitiv verarbeitet werden können, ohne dass eine Analyse der einzelnen Bestandteile notwendig ist. Sprachliche Strukturen sind als Form-Bedeutungs-Verbindungen Grundeinheiten der Kommunikation. Durch die kommunikative Verwendung wird die semantisch-pragmatische Ebene an die Form-Ebene gekoppelt (Behrens 2009: 431-432). Tomasello (2008) beschreibt diese Gebrauchsbasiertheit sprachlicher Form-Bedeutungs-Paare folgendermassen:

Wenn Menschen wiederholt ,ähnliche‘ Dinge in ,ähnlichen‘ Situationen sagen, entwickelt sich daraus mit der Zeit ein sprachliches Verwendungsmuster, das in den Köpfen der Benutzer als neue Kategorie oder Konstruktion schematisiert wird - mit unterschiedlichen Abstraktionsgraden. [...]

Es gibt keine sprachlichen Elemente - ob lexikalische oder syntaktische, konkrete oder abstrakte, reguläre oder idiomatische - die nicht [...] symbolisch sind; alle haben einen kommunikativen Bedeutungsgehalt, weil sie alle direkt aus dem Sprachgebrauch abgeleitet sind. Ein wichtiges Merkmal der Konstruktionsgrammatik ist, dass ihr Interesse Gebrauchsmustern jedweder Art gilt, d. h. auch solchen mit begrenzter Allgemeingültigkeit. Es wird also der Versuch unternommen, nicht nur die ,Kerngrammatik` zu erklären, sondern alle sprachlichen Elemente und Strukturen - einschließlich idiomatischer Ausdrücke, irregulärer Muster, gemischter Konstruktionen und metaphorischer Extensionen - und zwar im Rahmen einer einzigen Theorie. (Tomasello 2008: 21)

Unterstrichen wird also die Bedeutung der Frequenz für die Herausbildung von Konstruktionen: Durch das wiederholte Verwenden einer bestimmten Struktur 
Tab. 6.1: Beispiele für Konstruktionen (Behrens 2011b: 381; Goldberg 2003: 220).

\begin{tabular}{ll}
\hline Konstruktion & Form/ Beispiel \\
\hline Morphem & zer-, ver-, anti-, -lich, -te \\
\hline Wort & Avocado, Anaconda, und \\
\hline komplexes Wort & Feuerteufel, sit-in \\
\hline fixiertes Idiom & Den Vogel abschiessen \\
\hline partiell fixiertes Idiom & In die X kriegen, wobei X = Wolle oder X = Haare \\
\hline Covariational conditional construction & je X desto $Y$ \\
\hline ditransitive Konstruktionen & $\begin{array}{l}\text { Form: Subj V Dat-Obj Akk-Obj / Er backte ihr einen } \\
\text { Kuchen }\end{array}$ \\
\hline Passiv & $\begin{array}{l}\text { Form: Subj werden (PP) Partizip Perfekt / Das Gürtel- } \\
\text { tier wurde von einem Auto überfahren }\end{array}$ \\
\hline
\end{tabular}

wird diese zu einem Verwendungsmuster, das mit unterschiedlichen Abstraktionsgraden schematisiert wird. Als Beispiele dafür führt Behrens (2011b: 381) mit Bezug auf Goldberg (2003: 220) Konstruktionen unterschiedlicher Abstraktheitsgrade auf: Das können etwa Morpheme, Wörter oder Schemata wie Je X desto $Y$ sein (vgl. Tab. 6.1).

Was Tomasello (2008: 21) im oben zitierten Ausschnitt ebenfalls betont, sind die kommunikative Funktion und der Sprachgebrauch, die Konstruktionen erst hervorbringen. Gerade die konsequente Orientierung am Sprachgebrauch macht aus dem konstruktionsgrammatischen Paradigma eine empirische Wissenschaft. Diese Ausrichtung an der Empirie vereint auch die unterschiedlichen konstruktionsgrammatischen Ausrichtungen. Was konstruktionsgrammatische Studien zusammenhält, so Ziem \& Lasch (2011: 275-276), ist keine gemeinsame Ausrichtung auf einen bestimmten Typ an Daten oder ein verbindliches Methodenrepertoire, sondern ein Konsens über eine Reihe von theoretischen und methodologischen Basisannahmen, die für die konkrete Forschungspraxis relevant sind:

- Sprache ist keine autonome Instanz, sie steht vielmehr in komplexer Interaktion mit anderen kognitiven Fähigkeiten, Ressourcen und anderen Zeichensystemen. Der Begriff der (generativen) Regel wird durch den des Kategorisierungslinks ersetzt, da jede Erzeugung von komplexen sprachlichen Ausdrücken bzw. Zeichen einen wissensbasierten Prozess darstellt.

- Grammatische Phänomene gehen aus Konzeptualisierungsprozessen hervor. Aus diesem Grund bilden Grammatik und Lexikon keine distinkten Einheiten, sondern ein Kontinuum, ein so genanntes ,Konstruktikon (Ziem \& Lasch 2011: 275-276). Sprachliches Wissen besteht aus diesem 
Konstruktikon, einem strukturierten Inventar an Konstruktionen (Fischer 2014: 243).

- Durch den Sprachgebrauch entsteht und verändert sich sprachliches Wissen. Die sprachlichen Strukturen resultieren aus Prozessen der kognitiven Verfestigung (entrenchment) und aus kognitiven Routinen. Grammatik ist daher ein Epiphänomen konkreter sozialer Interaktionsprozesse oder - mit Haspelmath (2002: 270) - „geronnener Diskurs“ (vgl. Kap. 3.2.3).

- Gelten diese drei Annahmen für die Kognitive Linguistik grundsätzlich, besteht das Spezifikum der Konstruktionsgrammatik darin, für die Beschreibung des Erwerbs, der Repräsentation sowie der Verarbeitung von sprachlichem Wissen die Konstruktion als grundlegende Einheit anzusetzen.

Konstruktionen haben damit auch eine psycholinguistische Relevanz. Ziem \& Lasch (2013: 77) nehmen eine erweiterte definitorische Begriffsbestimmung von Konstruktionen vor, die fünf für die Kognitive Linguistik insgesamt kennzeichnende Prinzipien (im Zitat hervorgehoben) aufführt, auf dem das auf den Sprachgebrauch ausgerichtete Forschungsprogramm der Konstruktionsgrammatik fusst:

Konstruktionen sind (a) nicht-kompositionelle und konventionalisierte Form-Bedeutungs-paare, die (b) kognitiv einen gestalthaften Charakter haben, gleichwohl aber (c) konstruierte Einheiten und als solche (d) konzeptueller Natur sind, insofern sie sich (e) kontextgebunden im Sprachgebrauch herausbilden und verändern. (Ziem \& Lasch 2013: 77, Hervorhebungen PS)

Die Definition repräsentiert den konstruktionsgrammatischen ,Mainstream', der sich insbesondere an den Arbeiten von Goldberg und Langacker orientiert (Ziem \& Lasch 2013: 77). Auer (2005) spezifiziert in seinen Ausführungen zum Konstruktionsbegriff in der Gesprächsanalyse zur Form von Konstruktionen mit Verweis auf Tomasello, sie seien „oft mehr oder weniger idiosynkratische Instantiierungen allgemeinerer syntaktischer Strukturen, die meist bestimmte interaktive Funktionen haben und die als solche im Erstspracherwerb noch vor den allgemeineren Strukturschemata gelernt werden“ (Auer 2005: 16). Er ergänzt die Definition damit um einen zentralen Erwerbsaspekt: Konstruktionen werden in der Sprachaneignung vor anderen Strukturschemata erworben. Besonders im Grad der Idiosynkrasie, so Auer, unterscheiden sich Konstruktionen;

manche Konstruktionen sind vollständig - auch lexikalisch - spezifiziert, andere lassen bestimmte Abänderungen zu oder enthalten Leerstellen und sind daher abstrakter. Per definitionem ist die Syntax und/oder die Semantik einer Konstruktion nicht kompositionell aus ihren Teilen ableitbar. Die kategorische Trennung zwischen Syntax und Lexikon löst sich auf. (Auer 2005: 16) 
Keine andere Grammatiktheorie stellt in Frage, dass es syntaktisch und/oder semantisch nicht-kompositionelle Strukturen gibt, sprachliche Ausdrücke also, deren Form und/oder Bedeutung sich nicht aus ihren Bestandteilen ableiten lässt. Die Bedeutung fester Formeln für den Spracherwerb ist daher bekannt. Aus der Vielzahl von Bezeichnungen dieses Phänomens hat sich insbesondere im DaZ-Erwerb etwa der Terminus Chunk etabliert (vgl. dazu etwa Heringer 2009). Alternativen aus der Spracherwerbsforschungen fassen Diehl et al. (2000: 340-341) unter folgenden Bezeichnungen und Paraphrasierungen zusammen: prefabricated patterns, formulaic expressions, chunk/schemata, formulaic knowledge, expressions of more or less formulaic and idiomatic character oder Formeln und Rahmen (vgl. für die Literaturangaben Diehl et al. 2000: 340341). Auch für den Erwerb formaler Strukturen auf Basis funktionaler Primate gibt es in der Forschung seit den 1980er Jahren verschiedene Beispiele. Behrens (2009: 432) verweist hierfür etwa auf Slobin (1985) oder auf Pinker (1989). Ihren Ansätzen ist gemeinsam, dass durch die Spracherfahrung die semantische Bindung syntaktischer Strukturen gelockert und der zielsprachlichen Varietät angeglichen wird. Es zeigte sich hingegen, dass formale Strukturen nicht zwingend funktional motiviert sein müssen oder in der frühen Kindersprache in funktional beschränkter Form verwendet werden. Kinder halten sich im Gegenteil von Beginn weg an die zielsprachliche Form-Funktions-Struktur (Behrens 2009: 432-433 mit Verweis auf Bowerman 1990). Kennzeichnend für die Konstruktionsgrammatik ist gerade diese Annahme sowie das Postulat, dass Konstruktionen das einzige Organisationsprinzip natürlichsprachlicher Grammatiken darstellen (Stefanowitsch 2011: 20). Darin unterscheidet sie sich von anderen Ansätzen, die nicht-kompositionelle Strukturen und ihre semantischpragmatische Funktion beschreiben.

Die theoretischen Prämissen der Konstruktionsgrammatik erfordern eine Orientierung am Sprachgebrauch sowie Evidenz für die psycholinguistische Realität der untersuchten Einheiten (Fischer 2014: 245). Empirisch ist daher für möglichst viele möglichst ,kerngrammatische‘ Strukturen (Stefanowitsch 2011: 20) nachzuweisen, dass es sich um Konstruktionen handelt. Wann ist eine komplexe Spracheinheit so idiomatisiert und verfestigt, dass von einer Konstruktion zu reden ist? Wie fest verankert müssen Konstrukte im Sprachwissen der Sprachteilhaber sein, um Konstruktionen darzustellen? Wie oft muss eine sprachliche Struktur als solche verwendet werden, um in den Status einer Konstruktion zu gelangen? In diesen Fragen ist die Theorienbildung der empirischen Forschung voraus.

Die Spracherwerbsforschung ist $\mathrm{zu}$ einem etablierten Anwendungsgebiet konstruktionsgrammatischer Untersuchungen geworden (Behrens 2009: 427). 
Aus diesen Forschungen werden Erkenntnisse gewonnen, die ihrerseits wiederum Eingang finden in die theoretische Ausgestaltung der Konstruktionsgrammatik und der Kognitiven Linguistik auf dem Weg hin zu einer umfassenden Sprach- und Kognitionstheorie. Der Blick auf konstruktionsgrammatische Spracherwerbsstudien zeigt eine ausgeprägte Konzentration auf frühe Erstspracherwerbssituationen, an die methodisch vorzugsweise korpuslinguistisch herangegangen wird. Im Zentrum empirischer Studien steht zudem oft nicht nur die Sprachproduktion des Kindes, sondern ebenfalls Struktur und Umfang des Inputs, beispielsweise in der Mutter-Kind-Interaktion. Offen bleibt die Tauglichkeit des konstruktionsgrammatischen Paradigmas für andere Spracherwerbsbereiche, darunter die Literalisierung und innerhalb der Literalisierung wiederum den Erwerb der (konzeptionellen) Schriftlichkeit.

\subsection{Literale Konstruktionen zwischen Prozess und Produkt des Schreibens}

Während laut Diessel (2008) von vielen Spracherwerbsforschern akzeptiert wird, dass sich die Konstruktionsgrammatik für die Analyse von frühen Zweiund Dreiwortäusserungen sehr gut eignet, wird das für komplexe grammatische Gebilde in Frage gestellt: Diese könnten „nicht mehr adäquat mit den Mitteln der Konstruktionsgrammatik beschrieben werden, und somit, so die Kritiker, sei der gesamte Ansatz als Modell für den Erwerb der Grammatik ungeeignet“ (Diessel 2008: 41). Diessel hingegen argumentiert, dass diese Kritik unbegründet sei, und zeigt am Erwerb von komplexeren grammatischen Gebilden,

\footnotetext{
dass sich komplexe Sätze aus einfachen, zumeist wortspezifischen Konstruktionen entwickeln. Dabei lassen sich zwei Entwicklungsverläufe unterscheiden: Der Erwerb von Komplement- und Relativsätzen lässt sich als ein Prozess der Satzexpansion beschreiben, während sich der Erwerb von Konjunktionalsätzen als ein Prozess der Satzintegration begreifen lässt. In beiden Fällen bilden einfache, nicht eingebettete Sätze den Ausgangspunkt für die Entwicklung von komplexen Satzkonstruktionen. (Diessel 2008: 53)
}

Er hebt auch hervor, dass sich konstruktionsgrammatische Ansätze dafür eigneten, die Kontinuität der Entwicklung von den ersten Einwortsätzen bis hin zu komplexen Satzkonstruktionen zu zeigen. „Was Kinder lernen, sind sprachliche Symbole, also Form-Funktionseinheiten, die sich lediglich in ihrer Komplexität und in dem Grad der Abstraktheit voneinander unterscheiden.“ (Diessel 2008: 53) Eine Grammatiktheorie, die sich - wie die Konstruktionsgrammatik - als Sprachtheorie versteht, muss Erwerb und Verwendung der 
Sprache insgesamt erklären können, um ihre Tauglichkeit nachzuweisen. Konstruktionsgrammatische Ansätze müssten daher auch auf den Erwerb des schriftlichen Ausdrucks im Rahmen von Fragen der Literalisierung anwendbar sein. Dabei rücken andere Aspekte und andere Konstruktionen ins Zentrum, und zwar speziell satzübergreifende Konstruktionen.

\subsubsection{Literale Prozeduren als transphrastische Konstruktionen}

Die Mehrzahl vorliegender konstruktionsgrammatischer Arbeiten geht über die Satzgrenze nicht hinaus (Feilke 2010: 2; Günthner 2009: 405). ${ }^{37}$ Das zeigen auch die von Goldberg (2003) zitierten Beispiele in Tabelle 6.1. Im 2015 erschienenen Band zu Konstruktionen im Spannungsfeld von sequentiellen Mustern, kommunikativen Gattungen und Textsorten von Bücker, Günthner \& Imo (op. 2015) wird dieses Desiderat aufgenommen und eine komplexe Musterhaftigkeit oberhalb der Satzgrenze in den Fokus genommen. Darin werden verschiedene Ansätze für die Suche nach transphrastischen Konstruktionen aus den Konzepten der Diskursmuster (insb. Östman op. 2015) und der Textlinguistik rezipiert und anhand empirischer Studien diskutiert. Mit keinem Beitrag vertreten ist in der Publikation trotz des expliziten Bezugs zur geschriebenen Sprache in einem separaten Abschnitt die Forschungstätigkeit zu literalen Prozeduren aus der Schreibforschung: Eine Gruppe von Forschenden um Helmuth Feilke führte (transphrastische ${ }^{38}$ ) schriftsprachliche Konstruktionen unter dem mittlerweile in der Schreibforschung breit rezipierten Begriff der literalen Prozedur, alternativ und synonym verwendet werden die Begriffe Textroutine oder Textprozedur, ${ }^{39}$ in die Diskussion ein. Literale Prozeduren stehen dabei als Chiffre „für den Fluchtpunkt einer Reihe schon länger aktueller Fragen in der Linguistik wie in der Sprachdidaktik " (Feilke 2010: 1). Sie stellen einen Ansatz dar, wie Konstruktionen als transphrastische Strukturen zu fassen sind, und zeigen, welche Rolle ihnen bei der Konstitution ganzer Texte zukommt. ${ }^{40}$ Aus

37 Das ergibt sich durch die Konzentration auf den frühen Spracherwerb, da hier Einwort-, Zweiwort- und Mehrwortäusserungen im Zentrum stehen.

38 Das Attribut transphrastisch bezeichnet Konstruktionen, die mehr als einen Satz umfassen. Beispielsweise sind das textstrukturierende Positionierungsprozeduren wie Auf der einen Seite ... Auf der anderen Seite.

39 Feilke hält den Terminus Textprozedur für am besten geeignet: „Er hebt mit ,Prozedur die Mittlerstellung zwischen dem Prozess- und dem Produktaspekt des Schreibens hervor und macht deutlich, dass es um eine auf Texte und deren Komponenten bezogene Kompetenz geht“ (Feilke 2014a: 11, Hervorhebung im Original).

40 Es ist zu vermuten, dass konstruktionsgrammatische Untersuchungen zur geschriebenen Sprache vorwiegend aus methodischen Gründen nicht Bezug nehmen auf die Forschungen zu 
der kompetenzorientierten Perspektive seien literale Prozeduren sprachliche Verfahren zur Textkonstitution:

[Das] Stabile von Prozeduren erkennt man an der wiederkehrenden Konstellation von Elementen. Sie können als Ausdrucksmuster schwach oder auch stärker grammatikalisiert sein und verbinden sich mit einem Gebrauchsschema. Literale Prozeduren sind eine didaktisch relevante Kategorie: Es können empirisch Grade der Beherrschung von Prozeduren unterschieden werden. Sie sind lern- und lehrbar. Der Erwerb ist kaum erforscht. (Feilke 2010: 1)

Feilke (2010: 2) illustriert am Beispiel von Überschriften die Merkmale von literalen Prozeduren: „Überschriften sind textkonzeptuelle Gestalten[,] [sie] haben eine besondere Syntax und Semantik, die sie vom Text abhebt[.]" Syntax und Semantik sind ,unter einer grammatischen Perspektive oft irregulär, was aber ihre Verstehbarkeit nicht beeinträchtigt“ (Feilke 2010: 2). Das wiederum sei ein Kennzeichen von Konstruktionen im Sinne der Konstruktionsgrammatik: „Konstruktionen sind prozedurale Formulierungsformate. Sie binden das syntaktische Muster an Handlungsaufgaben und erklären es darüber.“ (Feilke 2010: 2)

Als textkonzeptuelle Gestalt haben Überschriften eine ganz besondere Position im Text: Sie sind dem Text vorangestellt und von ihm visuell, aber auch sprachlich-strukturell durch eine spezielle Syntax und Semantik abgehoben (Feilke 2010: 2). Diese Position erklärt auch ihren Aufbau und gleichzeitig ihr Potenzial: Überschriften werden regelhaft verstanden, auch wenn sie oft grammatische Irregularitäten aufweisen, denn als Ausdrucksgestalten sind sie direkt einem Gebrauchsschema zugeordnet; „die konzeptuelle Gestalthaftigkeit von Überschriften als literalen Prozeduren besteht vor allem in einem der grammatischen Struktur vorgeordneten textuellen Verwendungs- und Interpretationsschema“ (Feilke 2010: 2). Überschriften zeigen deutlich, dass die grammatische Irregularität „sich als sogenannter Strukturverlust erst als mögliche Folge eines pragmatisch stabilisierten Gebrauchsschemas“ (Feilke 2010: 2) ergibt, und stellen gleichzeitig ein Beispiel für eine Konstruktion dar, die nur satzübergreifend und unter Mitberücksichtigung ihrer Funktion im konzeptionell schriftlichen Kontext zu erklären, zu verstehen und zu erwerben ist.

den literalen Prozeduren: Letztere zeichnen sich derzeit eher durch qualitative Analysen der Form und Funktion literaler Prozeduren aus und kaum durch einen quantitativen Zugang, wie er für konstruktionsgrammatische Arbeiten häufig gewählt wird. Gerade hierin ist ein wesentliches Potenzial für zukünftige Forschungen zu literalen Prozeduren zu sehen: Es wäre wünschenswert, an grossen Korpora datengeleitet zu ermitteln, welche (transphrastischen) Grössen das Potenzial für Konstruktionen im Sinne der Konstruktionsgrammatik haben. 
Literale Prozeduren sind das Ergebnis von Schrift- und Texterfahrung, sie können über das Memorieren von Ausdrucksmustern nicht vermittelt und gelernt werden. Das dafür notwendige Wissen bezeichnet Feilke (2010) als eine Kompetenz ,dritter Art', die als ein prozedurales Wissen zwischen dem Produkt- und dem Prozessaspekt der Sprache steht. Damit gemeint ist allerdings nicht ein Schreibprozesswissen, sondern ein textkonstituierendes sprachliches Wissen (Feilke 2010: 3). Literale Konstruktionen haben zwar prozessualen Charakter, weil sie die transphrastische und globale Textebene strukturieren und damit nicht nur den Text, sondern auch seine Aktualgenese segmentieren. Da sie aber ganz spezifische und idiomatisierte Strukturen darstellen, ist das Wissen über sie und ihre Verwendung immer sprachlich basiert. Für Feilke (2010) sind dann auch diese drei Merkmale literaler Prozeduren besonders zentral: Ihr prozessualer Charakter, ihre Sprachlichkeit und ihr transphrastisches Ordnungspotenzial. Literale Prozeduren seien immer lexikalisch ausgewiesen, da die Lexik einen ausdruckseitigen Anker aller darunterliegenden Ordnungsleistungen des Sprechens und Schreibens darstelle (Feilke 2010: 5). Sie stehen zwischen regelorientierter Konstruktion und dem Lexikon, sind „vielfach regulär gebildet, aber gleichermaßen als Ganze schematisch verfügbar, mit slots, die immer wieder auf andere Weise gefüllt werden können“ (Feilke 2010: 4). Die funktionale Prägung literaler Prozeduren liegt auf der textuellen Ebene, sie stehen zwischen der lokalen Ebene des einzelnen Satzes und der globalen des Textes (Feilke 2010: 5). Am Erwerb literaler Prozeduren zeigt sich zudem ein Phänomen, das der Spracherwerbsforschung bekannt ist: „Die formale Struktur einer syntaktischen oder auch textbildenden Routine wird erworben, bevor sie inhaltlich angemessen genutzt werden kann.“ (Feilke 2010: 15)

Hinter dem Konzept der literalen Prozedur steht wie hinter der Konstruktionsgrammatik ein dynamisches Sprachmodell, das annimmt, dass Strukturen gebrauchsbasiert angeeignet werden und sich wiederum auf das Sprachwissen auswirken. Eine Erweiterung des Konstruktionsbegriffs durch die Modellierung literaler Prozeduren ist darin zu sehen, dass transphrastische Strukturen in den Fokus rücken. Literale Prozeduren sind Textroutinen, die eine musterhafte semiotische Struktur aufweisen, über Kontextualisierungspotenzial verfügen und für den Aufbau von Schreibkompetenzen besondere Relevanz haben. Anhand literaler Prozeduren werden typische Teilhandlungen bestimmter Textsorten realisiert (Gätje, Rezat \& Steinhoff 2012: 127). Theoretisch mitgedacht sind zudem die Prozesshaftigkeit und der sprachbasierte Prozesscharakter von Konstruktionen: Gerade bei sprachlichen Strukturen mit transphrastischem Ordnungspotenzial, die beispielsweise Argumentationsvorgänge im Textprodukt strukturieren (einerseits- andererseits, zwar ... aber ...), erscheint es ein- 
sichtig, dass sie auch den Produktions-, also den Schreibprozess, rhythmisieren und segmentieren. Als schriftsprachliche und häufig vorkommende Routineformeln kommt ihnen im Formulierungsprozess eine kognitive Entlastungsfunktion zu (Gätje, Rezat \& Steinhoff 2012: 127).

Bei der Untersuchung literaler Prozeduren stehen bislang qualitative Herangehensweisen im Vordergrund, die ausgewählte sprachliche Prozeduren - denen Konstruktionscharakter unterstellt wird - in ihrer Funktion für die Schriftsprachaneignung fokussieren. Auf quantitative Auswertungen (vgl. dazu als Beispiel Gätje, Rezat \& Steinhoff 2012) wird zurückgegriffen, um (pseudolongitudinale) Aneignungsverläufe anhand der Frequenz bestimmter Prozeduren aufzuzeigen. Zur Didaktik von Textprozeduren liefern jüngere Forschungsergebnisse vielversprechende Ergebnisse. Eine Auswahl präsentieren Bachmann \& Feilke (2014). Besonders hingewiesen sei dabei auf die empirische Studie von Anskeit \& Steinhoff (2014): Sie untersuchen, wie sich die Partizipation an bestimmten Schreibarrangements auf die Textproduktion (Erstfassung, Revision und Endfassung) auswirkt, wobei davon ausgegangen wird, dass stärker profilierte Arrangements $\mathrm{zu}$ einer erfolgreicheren Textproduktion führen als schwächer profilierte. ${ }^{41}$ Erste Ergebnisse zeigen, dass insbesondere Schüler und Schülerinnen, deren Texte in sprachprofilierten Arrangements entstanden sind, von den entsprechenden Prozeduren häufiger Gebrauch machen und sie schemagerecht und kreativ anwenden. Den Kindern, die an sprachprofilierten Arrangements teilnahmen, wurden aufgabenspezifische Prozedurenausdrücke als sogenannte Formulierungstipps vorgelegt. Diese wurden vorgängig basierend auf der empirischen Studie von Augst et al. (2007) ausgewählt und entsprechen Ausdrücken, die sich für die Schreibentwicklung auf der Primarstufe als wichtig herausgestellt haben (Anskeit \& Steinhoff 2014: 139). Das Angebot an die Kinder, sich an vorgegebenen Prozeduren $\mathrm{zu}$ orientieren und diese in den eigenen Schreibprozess zu integrieren, erwies sich also als erfolgreich. Als unbegründet stellte sich hingegen die Befürchtung heraus, das Vorschlagen von Prozeduren führe zu einem ,pattern drill', einem schablonenhaften Schreiben (Anskeit \& Steinhoff 2014: 152).

Korpuslinguistisch untersucht wurden literale Prozeduren m. W. bislang nicht. Eine korpuslinguistische Studie, die für bestimmte Altersbereiche zentrale Konstruktionen herausstellt, wäre mit Sicherheit aufschlussreich.

41 Die Profilierung von Schreibaufgaben, als Konzept eingeführt von Bachmann \& BeckerMrotzek (2010), bezieht sich auf die Merkmale Funktion, Wissen, Interaktion und Wirkung, die in stark profilierten Aufgaben allesamt berücksichtigt werden. Vgl. zum Konzept der Profilierung Bachmann \& Becker-Mrotzek (2010); Bachmann \& Becker-Mrotzek (2011) sowie Kapitel 7.2.2 zur Beschreibung der Schreibaufgabe. 
Offen ist nämlich, ob Textprozeduren tatsächlich Konstruktionscharakter im Sinne konstruktionsgrammatischer Modellierungen haben. Was die Schreibforschung zur empirischen Klärung der Funktion von Textprozeduren beitragen könnte, betrifft die Schnittstelle zwischen Textproduktion und Textprodukt: Wenn literalen Prozeduren unterstellt wird, als Bindeglied zwischen Textprodukt und Textprozess besondere Relevanz zu haben, dann ist das Medium ihrer Produktion, also das Schreiben, eigentlich dafür geeignet zu untersuchen, ob sich ihre Ausformulierung im Schreibprozess verfolgen lässt. Möglicherweise strukturieren sie als Konstruktionen Sprache nicht nur kognitiv, sondern auch in der Produktion (vgl. dazu Kapitel 8.1.2 zu den Schreibsalven).

\subsubsection{Literale Konstruktionen als Regulativ im Schreibprozess}

Schreibroutine zeigt sich nicht nur verfestigt in Textprodukten, sondern auch während des Schreibprozesses. Sie ist das Ergebnis von Schreiberfahrung und Resultat einer sozialen Praxis, die durch Teilhabe an (schriftlicher) gesellschaftlicher Kommunikation und literalem Handeln entsteht. Wie sich aus Literalitäts- und Schreiberfahrungen konkrete Textprozeduren herausbilden respektive was dabei auf der kognitiven Ebene vor sich geht, ist empirisch und theoretisch nicht geklärt. In den Schreibprozessmodellen etwa von Hayes und Flower kommt die Schreiberfahrung als text produced so far vor (vgl. Kap. 4.2). Dieser und Aspekte aus der Planung gehen, so Hayes (1996: 23-24), in den Schreibprozess ein:

Cues from the writing plan and from the text produced so far are used to retrieve a package of semantic content. This content is stored in working memory but not in the articulatory buffer. [...] A surface form to express this content is then constructed and stored in the articulatory buffer. [...] When all of the content is expressed or when the capacity limit of the articulatory buffer is reached, the sentence part is articulated either vocally or subvocally. If all of the current content has been expressed, then the writer may show evidence of searching for new content. If the articulated sentence part is positively evaluated, then it is written down and the process is repeated. If it is rejected, a new sentence part is constructed and evaluated.

Schreiberfahrung und grundsätzlich Spracherfahrung reduzierten die Belastung des Arbeitsgedächtnisses bei der Überführung von Inhalten in ,sentence parts‘ (Hayes 1996: 23). Daraus könnte geschlossen werden, dass mit zunehmender Schreiberfahrung diese ,sentence parts‘ umfangreicher werden. Das wiederum könnte sich in Schreibprozessen zeigen (vgl. dazu die Ausführungen $\mathrm{zu}$ den Schreibsalven in den Kapiteln 8.1.2, 9.1.5 sowie 11.1.3).

Im Anschluss an die Ausführungen zur Konstruktionsgrammatik stellt sich die Frage, was genau „the surface form to express [semantic] content“ und 
dann die „sentence parts“ (Hayes 1996: 23) darstellen. Folgt man dem Konzept der Konstruktionsgrammatik, die von grammatischen Strukturen ausgeht, die psychologisch real respektive psychologisch realistisch sind (Stefanowitsch 2011: 11-12), liessen sich diese ,sentence parts“ als Konstruktionen interpretieren. Konstruktionen sind Schlüsselelemente zwischen dem Produkt- und dem Prozessaspekt der Sprache und als Regulativ im Schreiben in den von Hayes beschriebenen Teilprozessen bereits mitgedacht, auch wenn sie nicht präziser ausgeführt oder begrifflich spezifiziert werden. Um ganz konkrete sprachliche Konstruktionen geht es in der Modellierung bei Hayes nicht, das Modell wäre allerdings an dieser entsprechenden Stelle erweiterbar um literale Prozeduren und sprachliche Routinen, und zwar sowohl in ihrer sozialen als auch individuellen Dimension: Die Texte produced so far stellten die Schreiberfahrung dar, die in spezifischen sozialen Kontexten angeeignet wird und die sich als Übersetzung von Inhalt in konkrete sprachliche Strukturen im Moment des Niederschreibens äusserte. Ein methodischer Vorteil von Untersuchungen zur Schreibaneignung liegt in der Möglichkeit, Schreibprozesse sicht- und auswertbar zu machen. Sie zeigen, wie sprachliche Einheiten produziert werden, und machen Schreibroutine empirisch mess- oder zumindest beschreibbar.

\subsection{Literale Konstruktionen zwischen Text und Sprachwissen}

Die Konstruktionsgrammatik folgt in vielem einer für moderne Grammatiktheorien seit de Saussure charakteristischen Annahme: Sprache wird als prinzipiengeleitetes System verstanden, „das sich aus miteinander korrelierenden Elementen zusammensetzt, deren Ort im System sich zwar ändern kann [...], ohne dabei aber die strukturelle ,Ordnung' des sprachlichen Wissens generell zu gefährden“ (Ziem 2014: 15-16). Allerdings unterscheidet sie sich in ihren Erkenntnisinteressen von anderen Grammatiktheorien insofern fundamental, als sie auf ein Modell grammatischer Strukturen abzielt, das psychologisch real (oder zumindest psychologisch realistisch) ist. „Sie versteht sich deshalb primär als eine Theorie grammatischen Wissens und nur sekundär als eine Theorie grammatischer Systeme“ (Stefanowitsch 2011: 12). Da die Konstruktionsgrammatik sprachliches Wissen als Inventar von Form-Bedeutungspaaren und damit als eine Art Netz von Konstruktionen - ausgedrückt im Terminus Konstruktikon ansieht, stellt sich die Frage, in welcher Verbindung die einzelnen Konstruktionen zueinander stehen. In Bezug auf den Spracherwerb besonders zentral ist die Frage, ob die Relationen zwischen Konstruktionen dergestalt sind, dass einige Konstruktionen den Erwerb von anderen stützen und erleichtern können (vgl. zu dieser Frage Fischer 2014). Über die Struktur des Konstruktikons ist 
derzeit relativ wenig bekannt (vgl. aktuelle Forschungen bei Ziem 2014; Ziem \& Ellsworth 2016; Lasch \& Ziem 2014), insbesondere empirische Untersuchungen sind noch rar. Die unterschiedlichen Ausrichtungen innerhalb der Konstruktionsgrammatik unterscheiden sich zudem gerade darin, von welchen Verbindungen zwischen Konstruktionen sie ausgehen. Grundsätzlich sind Beziehungen verschiedener Natur möglich: Da Konstruktionen Form-Bedeutungspaare sind, können sich Verbindungen zwischen ihnen sowohl auf formale als auch auf semantische oder pragmatische Aspekte beziehen. Ebenfalls kann die Beziehung durch funktionale Synonymie oder durch Kookkurrenz in ähnlichen Situationen beruhen (Fischer 2014: 243-244).

\footnotetext{
Sprache und somit auch Grammatik wird immerfort geschaffen. Die zielorientierte Kreativität des Sprechers setzt freilich an den oberen grammatischen Ebenen an, wo er die Freiheit zum Manipulieren hat. Des Sprechers unmittelbares Ziel ist es, expressiv zu sein. Dadurch überlagert er immer wieder schon vorhandene Ausdrucksmittel, deren Einsatz sich automatisiert. Dadurch entsteht Grammatik. [...] Sie wird also nicht als eigenes Ziel angestrebt, sondern ist das notwendige Ergebnis der Neugestaltung von Ausdrucksformen auf den höheren Ebenen. (Lehmann 1995: 1265)
}

In diesem Aspekt decken sich konstruktionsgrammatische Ansätze mit der Grammatikalisierungstheorie. Sprachliches Wissen und Sprachhandlungswissen sind in dieser Perspektive miteinander verbunden, indem das eine nicht die Ressource für das andere darstellt, sondern beide Aspekte als Ressource und als Ergebnis des Sprachgebrauchs angenommen werden.

Strukturen mit kognitiver oder psychologischer Qualität haben mit der Repräsentation und mit der Wahrnehmung sprachlicher Strukturen zu tun. Konstruktionen stellen als sprachliche Strukturen Sinn- und Wahrnehmungsgestalten dar, wie am Beispiel der Überschriften gezeigt wurde. Sie sind textkonzeptuelle Gestalten, die sich durch die Syntax und die Semantik, aber auch durch die initiale Position im respektive vor dem Text abheben. Mit dem Schreibprozess einher geht eine Visualisierung, denn Texte präsentieren sich immer mit einer bestimmten äusseren Form: Sie sind nicht nur lesbare Gebilde, sondern solche mit einem visuellen Charakter. Überschriften zeigen exemplarisch, wie sprachliche Konstruktionen sich auch durch solche visuelle Aspekte auszeichnen. Im Fall der Überschrift handelt es sich dabei um die optische Absetzung vom Text durch das Voranstellen der Überschrift. Die Ausdrucksseite von Überschriften ist damit im doppelten Sinne salient, wie mit Bezug auf Feilke (2010) gezeigt wurde (vgl. Kap. 6.3.1); einerseits durch die strukturell routinehafte Formulierung, andererseits durch ihr visuelles Erscheinen. ${ }^{42}$ Das

42 Auf die Frage, wie Textprozeduren zwischen Erwerb und Nutzung von sprachlichem Wissen vermitteln, geht Rezat (2014) ein: Sie greift dafür auf den Ansatz der kognitiven Ergonomie 
mag für Überschriften in besonderem Mass gelten, dürfte aber auch für andere sprachliche Konstruktionen im Erwerbsprozess von Bedeutung sein. Es liesse sich sogar die Annahme formulieren, dass gerade für Kinder visuelle Aspekte sprachlicher Konstruktionen von besonderer Relevanz sind. Wie der erste Zugang zur Schrift anhand einzelner Grapheme die Übersetzung eines Lauts in ein visuelles ,Bild‘ darstellt, könnten visuelle Merkmale auch das Verfassen und Strukturieren ganzer Texte prägen. Die Auswertungen der Kinderkommentare im Rahmen der vorliegenden Studie werden diese Fragen wiederaufnehmen (vgl. Kap. 11.2.5).

\subsection{Zusammenfassung Kapitel 6}

„ich finde das nicht soooo eine gute idde“

Datenkorpus, ${ }^{43}$ Schülerin $4 \mathrm{w} 8$

Dynamische Modellierungen von Sprache stellen deren Gebrauch ins Zentrum. Grammatische Strukturen regeln diesen Sprachgebrauch, sind aber gleichzeitig auch dessen Ergebnis: Durch das Schreiben wird Sprachwissen angeeignet, ausgebaut, erweitert und restrukturiert. Die Routinenbildung, die dadurch zustande kommt, lässt sich konkret in der Genese literaler Prozeduren nachzeichnen: Diese werden im Verlauf der Ontogenese zunehmend literaler, also konzeptionell schriftlicher. Der oben zitierte Satz, der originalgetreu aus einem Kindertext aus dem Datenkorpus entnommen wurde, stellt eine Positionierungsprozedur dar, die Kinder zu Beginn der Schreibontogenese häufig verwenden und die später dann zugunsten anderer Konstruktionen in der Verwendungsfrequenz abnimmt (vgl. dazu die Datenauswertungen in Kap.11.3). Zudem aber enthält die Konstruktion einen Intensivierer, der aus einer konzeptionell mündlichen Ausdrucksweise stammt („Ich finde das nicht so eine gute Idee.“) und normwidrig verschriftet wird (,soooo“): Der Wunsch des Mädchens, die eigene Position über die literale Positionierungsprozedur zu verstär-

von Pierre Rabardel zurück (vgl. dazu Rabardel 1995, 1999). Das Konzept der kognitiven Ergonomie setzt die Annahme zentral, dass die Entwicklung höherer psychischer Funktionen auf eine vermittelnde Tätigkeit in Form von Werkzeuggebrauch angewiesen ist. Der Ansatz entstand in der kulturhistorischen Schule der 1920er und 1930er Jahre um Vygotskij, Lurija und Leontev, deren theoretischen Modellierungen in der Literalitäts- und Schreibforschung breit zitiert werden (darauf wurde im Zusammenhang mit Entwicklungsphasen des Schriftspracherwerbs und mit Einheiten metasprachlicher Reflexion in den Kap. 3 und 5 hingewiesen).

43 Der Ausschnitt stammt aus einem der in der vorliegenden Arbeit analysierten Briefe (vgl. dazu den Materialband). 
ken, findet in seinem Schreibrepertoire vorerst keine geeignete Entsprechung. Im Lauf der weiteren Textkompetenzaneignung werden sich die entsprechenden konzeptionell schriftlichen Mittel dafür erst noch ausbilden. Die kommunikative Notwendigkeit, einen Aspekt auch in der schriftlichen Varietät betonen und unterstreichen $\mathrm{zu}$ wollen, wird zunehmend Prozeduren generieren, die ohne mündliche Mittel (die Dehnung oder das lautere Aussprechen eines Wortes) auskommen. Das Ziel des Sprechers, so Lehmann (1995: 1265), „ist es, expressiv zu sein. Dadurch überlagert er immer wieder schon vorhandene Ausdrucksmittel, deren Einsatz sich automatisiert.“

Den Kern konstruktionsgrammatischer Ansätze bildet die Annahme, Konstruktionen als Form-Bedeutungspaare bildeten die einzige und grundlegende Einheit des Erwerbs, der Repräsentation sowie der Verarbeitung von sprachlichem Wissen. Letzteres wiederum wird darauf aufbauend als Netzwerk von Konstruktionen, als sogenanntes Konstruktikon, modelliert. Im Zentrum stehen damit sprachliche Strukturen, die nicht Kategorien des Sprachmodells darstellen, sondern solche, die einerseits Relevanz haben für die Sprachproduktion und Sprachaneignung und die andererseits psycholinguistisch real oder realistisch sind. Konstruktionen können mehr oder weniger idiomatisiert und mehr oder weniger umfangreich sein, was es ermöglicht, sprachliche Grössen verschiedener Art unter denselben Terminus zu subsumieren.

Die Schreib- und Schriftlichkeitsforschung ist für konstruktionsgrammatische Studien im Gegensatz zum frühen Spracherwerb bislang kein Hauptforschungsgebiet. Mit dem Konzept der literalen Prozeduren werden aktuell aber auch hier im Sinne der Konstruktionsgrammatik Konstruktionen und ihre Funktion für die Schriftsprachaneignung und spezifisch für die Textkompetenz fokussiert. Voraussetzung für die Verwendung literaler Prozeduren ist laut Feilke (2010: 3) ein Wissen dritter Art, ein textkonstituierendes sprachliches Wissen. Dabei handelt es sich - wie es die Grundlage für Konstruktionsgrammatiken und Grammatikalisierungstheorien bildet - um eine durch den Gebrauch angeeignete (usage-based) Routine, die sich konkret in sprachlichen Konstruktionen manifestiert. Da Konstruktionen und literale Prozeduren die realisierte Sprachstruktur und gleichzeitig ihre mentale Repräsentation darstellen, verbinden sie die Sprachprodukt(ions)- und die Sprachwissens- und Sprachprozessebene. Die Ansätze sind damit anschlussfähig an die Modellierung des Schreibprozesses, wie sie besonders prominent von Hayes und Flower vorgenommen und für kindliche Aneignungsprozesse u.a. von Berninger \& Winn (2008) differenziert wurde. Der Schreibprozess lässt sich zugänglich und visualisierbar machen und könnte darum eine neue Perspektive auf die Aktualgenese von Konstruktionen ermöglichen: Konstruktionen im Sinne der Konstruktionsgrammatik, aber auch literale Prozeduren stehen als mehr oder weni- 
ger idiomatisierte Strukturen zwischen dem Produkt- und dem Prozessaspekt der Sprache, zwischen kognitiver Repräsentation und sprachlicher Realisation. Der Weg von der mentalen Repräsentation zur Produktion sprachlicher Konstruktionen wird hier exemplarisch nachvollziehbar. Auch wenn das theoretisch einleuchtend ist, ist es doch empirisch noch schwer überprüfbar. Die computergestützte Schreibprozessforschung stellt zwar vielversprechende Methoden zur Segmentierung und Visualisierung des Schreibprozesses zur Verfügung, es bleibt aber dennoch offen, auf welchem Weg eine Konstruktion als solche identifiziert werden kann. Daher erhebt die vorliegende Studie nicht den Anspruch, eine konstruktionsgrammatische Analyse von Schreibprozessen liefern zu können. Eine grundsätzliche Ausrichtung an zentralen Merkmalen des konstruktionsgrammatischen Paradigmas hingegen erscheint für die vergleichende Analyse der Korpora zum Schreibprozess, zum Text und zu den metasprachlichen Kommentaren dennoch vielversprechend. Insbesondere geht es darum, auf den verschiedenen Ebenen Strukturen zu identifizieren, die als Form-Funktionspaare gelten dürfen und als solche produziert und von den Kindern wahrgenommen werden.

Die Einpassung des Konstruktionsbegriffs in den Erwerb von Textschreibkompetenz setzt die Erweiterung des Fokus auf transphrastische Konstruktionen voraus. Literale Konstruktionen werden zum Angelpunkt zwischen der lokalen Ebene des einzelnen Satzes und der globalen Textebene und erlangen dadurch textstrukturierendes Potenzial. Ihre psycholinguistisch reale Ausdrucksseite lässt zudem plausibel erscheinen, dass ihnen auch im Schreibprozess eine spezielle Rolle zukommt und sie auch darin zu identifizieren sind. Der Konstruktionsbegriff eignet sich damit für die vergleichende Auswertung der Teilkorpora der vorliegenden Studie: Diese nähert sich dem Prozesscharakter schriftsprachlicher Kindertexte einerseits über den Textentstehungsprozess und andererseits über Äusserungen der Kinder, die diese Eingriffe und die Textentstehung als Ganzes kommentieren. Die ausdrucksseitige Salienz, wie sie Feilke (2010) am Beispiel der Überschrift besonders augenfällig nachweist und die gerade in der kindlichen Modellierung von schriftsprachlichen Strukturen grundsätzlich Relevanz hat, könnte auch eine im Sprechen der Kinder über ihr Schreiben und über ihre Texte relevante Grössen sein. Die recalls könnten Aufschluss geben darüber, ob und wie Prozeduren bei Kindern auch im expliziten Sprachwissen verfügbar und abrufbar sind. Konstruktionen und literalen Prozeduren, die im Sinne der kognitiven Linguistik als „konzeptuelle oder mentale Entität [...] durch eine materielle, wahrnehmbare Entität (etwa Laute, Schriftzeichen, Gesten) symbolisiert [werden], so dass letztendlich die eine Erfahrung die andere evoziert“ (Langacker 2000: 5, zit. nach Behrens 2009: 431), könnte so auf die Spur gekommen werden. Im empirischen Teil 
wird der Versuch unternommen, anhand der Kinderkommentare aufzuzeigen, in welche Einheiten die Kinder ihre Sätze und Texte metasprachlich gliedern respektive auf welche sprachlichen Grössen sie ihre Kommentare beziehen: Sind das Kategorien wie Buchstaben, Wörter und Sätze oder andere zusammengehörige Einheiten im Sinne von Konstruktionen? 